\title{
UNSOLVED PROBLEMS
}

\author{
EARL J.TAFT
}

Let $A$ be the Hopf algebra $k[x], k$ a field, with $x$ primitive. The Hopf algebra dual $A^{0}$ is the space of linearly recursive sequences (B.Peterson and E.J.Taft, Aequationes Math. 20 (1980), 1-17). We gave an algorithm for diagonalizing a linearly recursive sequence in terms of the finite-dimensional subcoalgebra it generates. The product is that of divided-power series. Now let $W_{1}=\operatorname{Der} k[x]$, the Witt algebra. (Take characteristic $k \neq 2)$. The Lie algebra $W_{1}$ has basis $e_{i}=x^{i+1} \frac{d}{d x}$ for $i \geq-1$, with $\left[e_{i}, e_{j}\right]=(j-i) e_{i+j}$. W. Michaelis has recently shown that $W_{1}$ is a Lie bialgebra, with Lie coalgebra structure given by $\delta\left(e_{n}\right)=n\left(e_{n} \wedge e_{-1}\right)+(n+1)\left(e_{0} \wedge e_{n-1}\right)$, where $a \wedge b=a \otimes b-b \otimes a$ in $W_{1} \otimes W_{1}$. This seems to be related to the fact that $e_{0} \wedge e_{-1}$ satisfies the classical Yang-Baxter property in $W_{1} \otimes W_{1} \subseteq U\left(W_{1}\right) \otimes U\left(W_{1}\right)$. $W_{1}$ is a locally finite Lie coalgebra. Now $W_{1}^{0}$, the Lie coalgebra dual to the Lie algebra $W_{1}$, is also a Lie subalgebra of the convolution Lie algebra $W_{1}^{*}$, and $W_{1}^{0}$ is a Lie bialgebra. W. Nichols has recently shown that $W_{1}^{0}$ is the space of linearly recursive sequences.

Problem 1. Is there an algorithm to compute $\delta(f)$ in $W_{1}^{0} \otimes W_{1}^{0}$ for $f$ in $W_{1}^{0}$ ? This is easy if $f=e_{n}^{*}$ in the dual basis, i.e., for the finite sequences. $W_{1}^{0}$ is not locally finite as a Lie coalgebra, so this may not be analogous to $\Delta f$ in $A^{0} \otimes A^{0}$.

Problem 2. Is there any relation between the (commutative, cocommutative) Hopf algebra $A^{0}$ and the Lie bialgebra $W_{1}^{0}$ (both of which are the space of linearly recursive sequences)? Since everything starts from $k[x]$, there should be some relation between the two structures.

Problem 3. Is there a quantum deformation of any of these structures - $A, A^{0}, W_{1}$ and $W_{1}^{0}$ ? 